\title{
High latitude magnetospheric topology and magnetospheric substorm
}

\author{
E. E. Antonova ${ }^{1,2}$, I. P. Kirpichev ${ }^{1,2}$, I. L. Ovchinnikov ${ }^{1}$, K. G. Orlova ${ }^{1}$, and M. V. Stepanova ${ }^{3}$ \\ ${ }^{1}$ Skobeltsyn Institute of Nuclear Physics Moscow State University, Moscow, Russia \\ ${ }^{2}$ Space Research Institute RAS, Moscow, Russia \\ ${ }^{3}$ Physics Department, Universidad de Santiago de Chile, Santiago, Chile
}

Received: 8 September 2008 - Revised: 21 October 2009 - Accepted: 21 October 2009 - Published: 28 October 2009

\begin{abstract}
This study is focused on the problem of the localization of substorm expansion onset. In this context, the high latitude topology of transverse magnetospheric currents has been analyzed. This study has included the radial distribution of plasma pressure near noon, obtained using the THEMISB satellite data, the daytime compression of magnetic field lines and the existence of magnetic field minima far from the equatorial plane, given by all geomagnetic field models. As a result, the dayside integral transverse currents at the geocentric distances $7-10 R_{E}$ has been estimated. It is suggested, that nightside transverse currents at geocentric distances $\sim 7-$ $10 R_{E}$ are closed inside the magnetosphere and with dayside transverse currents form surrounding the Earth current system (cut ring current or CRC) which topologically is the high latitude continuation of ordinary ring current. A possibility of localization of substorm expansion onset at the nighside CRC region is analyzed using the experimental evidences that the onset is localized at geocentric distances $<10 R_{E}$.
\end{abstract}

Keywords. Magnetospheric physics (Current systems; Magnetospheric configuration and dynamics; Storms and substorms)

\section{Introduction}

The problem of classical isolated substorm expansion onset is deeply connected to the dynamics of magnetospheric current systems. Akasofu (1964) showed that the substorm expansion onset starts with the nearest to the equator auroral arc brightening or with the formation of a new arc in this region. A posteriori, this result has been confirmed by numerous results (see Frank and Sigwarth, 2000; Lyons et al.,

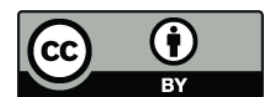

Correspondence to: E. E. Antonova (antonova@ orearm.msk.ru)
2002; Dubyagin et al., 2003; Lui, 2004; Zou et al., 2009; etc.). Localization of an isolated substorm expansion onset is one of the main endeavors of the THEMIS satellite mission. Lui et al. (2008) have found that the onset is localized at the geocentric distances $<10 R_{E}$, confirming previous results (see also the discussion in the paper Antonova et al., 2009a).

In this paper, we analyze the topology of transverse high latitude magnetospheric currents, and its importance for localization of the substrom expansion onset. We evaluate transverse currents near noon using data obtained by THEMIS satellite mission and show that such currents can be closed inside the magnetosphere by transverse currents at the same geocentric distances. According to the common point of view, the first auroral arc brightening is a result of tail current dynamics. We try to show that this statement can be reanalyzed assuming that transverse currents at geocentric distances $<10 R_{E}$ are probably closed not by magnetopause currents but currents inside the magnetosphere.

\section{Plasma domain surrounding the Earth and the region of quasi trapping}

Existence of plasma sheet like plasma domain surrounding the Earth became clear after the publication of the paper of Newell and Meng (1992), in which they showed that plasma sheet particle precipitations near noon come from a region situated at the equator from the low latitude boundary layer. Starkov et al. (2002) verified the picture obtained by Newell and Meng (1992). OVATION model (Newell et al., 2002) based on extended set of the DMSP particle precipitation data also shows the existence of closed ring of plasma sheet like precipitations. Vorobjev et al. (2007) supported this result. They showed the existence of a closed loop structure of plasma sheet precipitations under all geomagnetic conditions. 


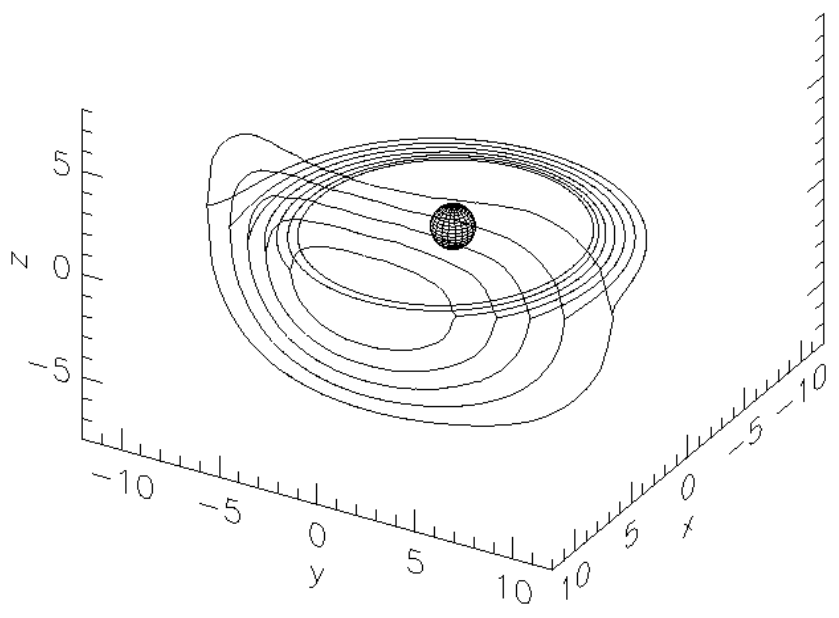

Fig. 1. The configuration of $B=$ const isolines, calculated using Tsyganenko-2004 model and solar wind parameters $P_{S W}=2 \mathrm{nPa}$, IMF $B_{Z}=-5 \mathrm{nT}, B_{Y}=B_{X}=0$ and zero inclination of Earth's magnetic dipole.

Figure 1 shows an example of the calculation of isolines of minimal values of the magnetic field at the magnetic field line using one of the latest models of Tsyganenko - Tsyganenko-2004 (Tsyganenko and Sitnov, 2005). Solar wind dynamic pressure $P_{S W}=2 \mathrm{nPa}$, IMF parameters $B_{Z}=-5 \mathrm{nT}, B_{Y}=B_{X}=0$ and zero inclination of Earth's magnetic dipole are selected as input parameters, to reproduce the geomagnetic field under typical solar wind conditions avoiding distortions related to the tilt effects. The first curve corresponds to the magnetic field in the internal ring current $(100 \mathrm{nT})$. The following curves are traced for the values decreasing in $10 \mathrm{nT}$ from the curve to the curve up to $40 \mathrm{nT}$ (external curve). Isolines with magnetic field smaller than $30 \mathrm{nT}$ begin to cross magnetopause that could be related to the adequate limit of use of Tsyganenko-2004 model. It is necessary to stress, that the majority of existing models of the magnetospheric magnetic field reproduces the same topology of the field lines: The magnetic field minima are shifted from the equatorial plane near noon.

Shown on Fig. 1 structure of the magnetic field determines the drift trajectories of energetic particles. Region from the geostationary orbit till $\sim 10 R_{E}$ was named the region of quasi trapping at the first stages of magnetospheric studies. From that time, it is well known that energetic particle trajectories cross the magnetopause when the particle pitch angle is equal to $90^{\circ}$. On the other side, drift shell splitting effect is observed for particles with smaller pitch angles (Shabansky and Antonova, 1968). Drift trajectories of such particles are closed inside the magnetosphere (see, for example, the discussion in the papers (Delcourt and Sauvaud, 1999; Öztürk and Wolf, 2007)). In this context, the appearance of the drift echo that is one of the constantly observed features of magnetospheric substorm, indicate the necessity of careful analysis of the topology of transverse currents. In particular, Hori et al. (2003) showed the existence of the effect of the drift echo until geocentric distances $\sim 12-13 R_{E}$ near midnight, using results of Geotail observations. This fact indicates that the region of the quasi trapping is localized at geocentric distances from $\sim 7 R_{E}$ till $\sim 12-13 R_{E}$, i.e. at the distances for which the transverse currents are ordinarily assumed to be closed by the currents at the magnetopause.

The latest model of external geomagnetic field Tsyganenko - TS07 (Tsyganenko and Sitnov, 2007; Sitnov et al., 2008) was developed using large sets of spacecraft data and represents the geomagnetic field generated by currents localized near the equatorial plane. Tsyganenko and Sitnov (2007), Sitnov et al. (2008) showed that fully developed ring current extends quite far down the tail (through $X_{\mathrm{GSM}} \sim-10 R_{E}$ ) and argued that it is a combination of the partial ring current closed through Region 2 field-aligned currents of Iijima and Potemra and the enhanced tail current closed via the magnetopause on both dusk and dawn sides. It is necessary to stress that all transverse currents in TS07 model are localized at the equatorial plane. Nevertheless, the location of the magnetic field minima at high latitudes at the daytime magnetic field lines shows the possibility of existence of comparatively large transverse currents near noon far from the equatorial plane. In this case, nighttime currents can be closed by such currents forming a surrounding the Earth current ring. This current system, named the cut ring current (CRC), was introduced by Antonova and Ganushkina (2000) as the daytime part of this ring splits into two branches (see also Antonova, 2003, 2004, for details).

\section{Daytime transverse current in the conditions of mag- netostatic equilibrium}

Localization of magnetic field minima far from the equatorial plane shows that the determination of the current distribution at the daytime field lines requires an analysis of data obtained far from the equatorial plane by a number of satellites. Such data are not available now. However, values of current densities and integral transverse current can be estimated assuming the validity of the condition of magnetostatic equilibrium when distribution of plasma pressure is nearly isotropic, that is observed at large geocentric distances (see DeMichelis et al., 1999). In such a case, transverse current $\boldsymbol{j}_{\perp}$ is equal

$\boldsymbol{j}_{\perp}=\boldsymbol{B} \times \nabla p / B^{2}$

where $\nabla p$ is the plasma pressure gradient, $\boldsymbol{B}$ is the magnetic field value. Equation (1) indicates that surrounding the Earth plasma domain contains transverse westward current when the plasma pressure gradient has the earthward direction.

Taking into consideration that the plasma pressure has a constant value along a field line in accordance with the condition of magnetostatic equilibrium, it is possible to evaluate 
current density at any point of a field line, if plasma pressure distribution at the equatorial plane is known, using one of magnetic field models. Although such approach is not self consistent, it makes it possible to estimate current densities far from the equatorial plane.

Both statistical and event-oriented studies of the plasma pressure distribution in the inner magnetosphere has been done during last two decades. In particular, Lui and Hamilton (1992), DeMichelis et al. (1997, 1999), Millino et al. (2001) obtained the distribution of plasma pressure at the equatorial plane using data of AMPTE/CCE observations. The global picture of magnetospheric plasma pressure distribution at $L<9$ demonstrates the presence of nearly azimuthally symmetric plasma distribution at $L \sim 7-9$ (DeMichelis et al., 1999). Such distribution supports the existence of a plasma domain surrounding the Earth. The plasma pressure gradient in this domain has an earthward direction, which implies the existence of a westward transverse current. DeMichelis et al. (1999) reproduced current density in the equatorial plane using values of plasma pressure gradients derived from AMPTE/CCE satellite and the Tsyganenko-87 (Tsyganenko, 1987) magnetic field model. The nighttime part of the obtained by DeMichelis et al. (1999) picture corresponds to comparatively large current densities $\sim 3-5 \mathrm{nA} / \mathrm{m}^{2}$ and was attributed to the partial ring current. Daytime current densities were smaller $\sim 1 \mathrm{nA} / \mathrm{m}^{2}$. These values can be used for the estimation of integral transverse current near noon only at the geocentric distances smaller than $7 R_{E}$ where field lines are not compressed. Antonova et al. (2009b) estimated daytime current densities using data of AMPTE/CCE and showed that the integral daytime current is comparable with the nighttime current at the same geocentric distances.

Obtaining daytime integral transverse current requires the analysis of magnetic field distribution along a field line. It is necessary to mention also that a significant part of the transverse current can be concentrated at a geocentric distance larger than the apogee of AMPTEE/CEE. Therefore, it may be interesting to extend the analysis of the dayside radial plasma pressure gradients till the magnetopause.

Such possibility appeared with the beginning of the operation of THEMIS satellite system. Orbits of 5 satellites of THEMIS are located near to the equatorial plane which gives the possibility to restore the radial distribution of plasma pressure. To obtain the first estimations of radial plasma pressure gradients we use the results of THEMIS-B satellite observations for the period 2 June 2007-29 October 2007 (http://www.nasa.gov/mission_pages/themis/) at the equatorial plane near noon. Parts of trajectories were selected at the geocentric distances $7<r<12 R_{E}$ with limitation of the azimuthal angle in $\pm 20^{\circ}$. The distribution of plasma pressure inside the magnetosphere near noon can be greatly influenced by the solar wind conditions. To take into account this dependence special algorithm of obtaining averaged plasma pressure profile is developed. Data of Wind satellite are used for the determination of the solar wind dynamic pres-

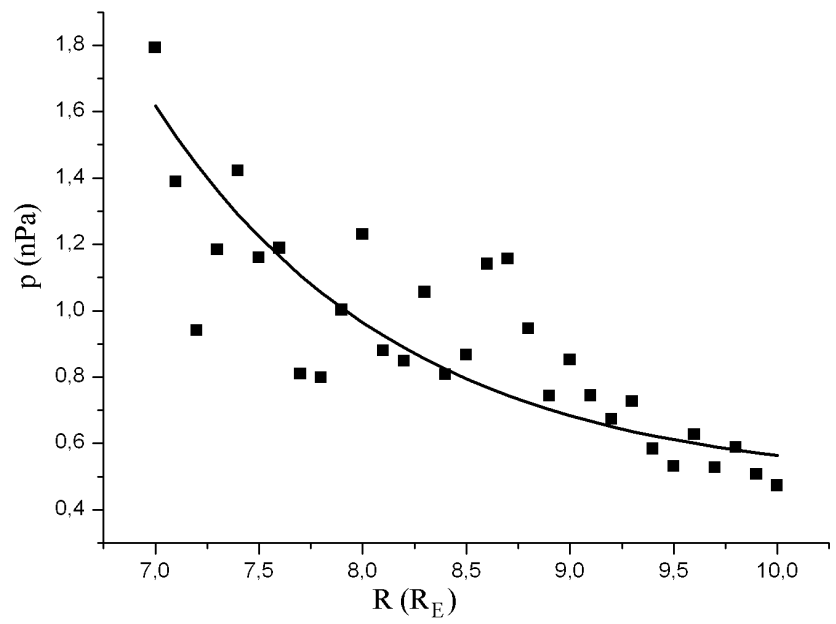

Fig. 2. Dayside radial plasma pressure profile obtained using data of THEMIS-B observations and its approximation used for the calculation of current densities.

sure $P_{s w}$ and Z-component of the interplanetary magnetic field. The Shue et al. (1997) model is used for the determination of the magnetopause location for every THEMIS-B measurement used. The averaged solar wind dynamic pressure $-P_{s w}=2.5 \mathrm{nPa}$ and averaged IMF $B_{Z}=-5 \mathrm{nT}$ were selected for the determining the average magnetopause position. The radial distance for the subsolar point in such a case is equal to $9.8 R_{E}$. All coordinates of satellite THEMIS-B are normalized to this position by linear compression or expansion. All obtained values are distributed along $X$ axes with the bin equal $0.5 R_{E}$ and averaged inside each bin. Figure 2 shows the obtained averaged radial plasma pressure profile (dots) fitted by an exponent (solid line). The geomagnetic field configuration at the dayside corresponding to the same values of the solar wind dynamic pressure and the IMF $\left(P_{s w}=2.5 \mathrm{nPa}\right.$, averaged IMF $B_{Z}=-5 \mathrm{nT}, B_{y}=0$, and $D_{s t}=-5 \mathrm{nT}$ ) has been obtained using Tsyganenko-01 model (Tsyganenko, 2002a, b).

Figure 3 shows the position of minima of the magnetic field at the daytime magnetic field lines. The values of current densities obtained by applying the relation (1) to the data of radial plasma pressure distribution shown in Fig. 2 are given in the regions of magnetic field minima. Calculated current densities at any point of field line give the possibility to evaluate the integral transverse current at the geocentric distances starting from 7.5 $R_{E}$ till the magnetopause in both hemispheres. It is also necessary to take into account that daytime currents are spread at larger areas than nighttime currents. For selected parameters, integral current constitutes $5.8 \times 10^{5} \mathrm{~A}$ in both hemispheres. This value is in agreement with estimations using plasma pressure profiles obtained by Lui and Hamilton (1992), DeMichelis et al. (1997, 1999) for the same selected parameters of Tsyganenko-2001 model and with the results obtained by Antonova et al. (2009b). The 


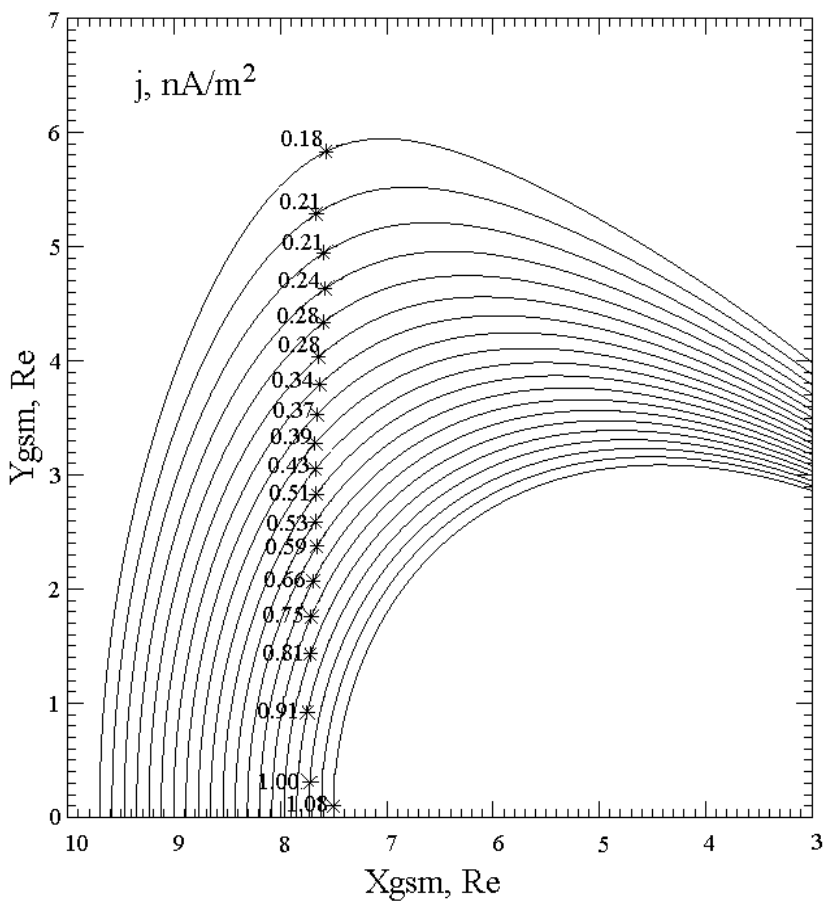

Fig. 3. Positions of minima of the magnetic field at the daytime magnetic field lines and calculated transverse current densities in the regions of magnetic field minima.

center of transverse currents situated at the dayside field-lines is deposited at $X_{\text {eff }}=7.3 R_{E}, Z_{\text {eff }}=2.7 R_{E}$. It is possible to see analyzing near Earth tail current distribution in Tsyganenko quite time models that considerable part of tail current can be closed inside the magnetosphere by dayside transverse currents.

\section{Conclusions and discussion}

Our analysis shows that traditional interpretation of the nighttime magnetospheric region at geocentric distances from 7 till $\sim 10-12 R_{E}$ as a tail region may be corrected. It was shown that comparatively large transverse current can be generated in the daytime magnetosphere under an assumption of magnetostatic equilibrium. To make this estimation, radial profiles of plasma pressure gradients have been obtained using the THEMIS-B satellite data, and the dayside configuration of the geomagnetic field has been obtained using the Tsyganenko-01 geomagnetic field model. Transverse currents are generated when the plasma pressure gradients are directed to the Earth. The obtained value of integral transverse current in the dayside is near to known transverse current values at the nightside magnetosphere at the same geocentric distances. This fact supports the assumption made by Antonova and Ganushkina (2000), Antonova (2003, 2004) about the existence of high latitude continuation of the ordinary ring current split into two branches in the dayside mag- netosphere, named the cut ring current (CRC). Topologically, this current is the high latitude continuation of ordinary ring current, and it is generated by plasma pressure gradients directed to the Earth. Nevertheless, the verification of such suggestion requires also the analysis of global plasma pressure distribution, which will be possible to do in the future. It is necessary to stress, that the Tsyganenko geomagnetic field models consider the transverse currents inside the magnetosphere localized near the equatorial plane. Therefore the existence of additional transverse current system far from equatorial plane can explain some difficulties of these models.

Obtained results also may be used for possible reanalysis of the position of the region of isolated substorm expansion onset. The equatorial boundary of the auroral oval is mapped at geocentric distances smaller than $\sim 10 R_{E}$. This can mean that this boundary is localized in CRC region. Significant number of observations shows that the first auroral arc brightening is localized near the equatorial boundary of the auroral oval. Correspondingly, this can mean that the first auroral arc brightening can be localized in CRC region. Naturally, such suggestion can be verified only after the appearance of the model of the magnetic field, which takes into account the existence of the daytime transverse current far from the equatorial plane. The validity of our suggestion can lead to revision of the analysis of the processes of substorm expansion onset. Theories of plasma instabilities for substorm expansion onsets discussed by Lui (2004) can be modified taking into account their possible development inside the CRC region. The support of the theory of substorm expansion onset suggested by Stepanova et al. (2000) will require to find the explanation how multiple inverted $\mathrm{V}$ structures are formed in $\mathrm{CRC}$ region, etc. All these problems will be the subject for future study.

Presented material demonstrates the possibility to reanalyze some important features of substorm dynamics. This does not mean that obtained earlier results are incorrect. It is only a contribution to the elaboration of the unified selfconsistent picture of the substrom dynamics, which will include all previous findings.

Acknowledgements. We thank the teams who provided THEMIS and Wind data obtained via NSSDC CDAWEB online facility. Special thanks to the Themis SSL Berkeley data service (V. Angelopoulos) for magnetic field (FGM, K. H. Glassmeier, U. Auster and W. Baumjohann), and plasma (ESA, C. W. Carlson and J. P. McFadden) data. The work is supported by FONDECYT grant 1070131, RFBR grants.

Topical Editor R. Nakamura thanks V. Vorobjev and another anonymous referee for their help in evaluating this paper.

\section{References}

Akasofu, S.-I.: The development of the auroral substorm, Planet. Space Sci., 12(4), 273-282, 1964. 
Antonova, E. E. and Ganushkina, N. Yu.: Inner magnetospheric currents and their role in the magnetosphere dynamics, Phys. Chem. Earth (C), 25(1-2), 23-26, 2000.

Antonova, E. E.: Investigation of the hot plasma pressure gradients and the configuration of magnetospheric currents from INTERBALL, Adv. Space Res. 31(5), 1157-1166, 2003.

Antonova, E. E.: Magnetostatic equilibrium and current systems in the Earth's magnetosphere, Adv. Space Res. 33(5), 752-760, 2004.

Antonova, E. E., Kornilov, I. A., Kornilova, T. A., Kornilov, O. I., and Stepanova, M. V.: Features of auroral breakup obtained using data of ground-based television observations: case study, Ann. Geophys., 27, 1413-1422, 2009a, http://www.ann-geophys.net/27/1413/2009/.

Antonova, E. E., Kirpichev, I. P., Stepanova, M. V., Orlova, K. G., and Ovchinnikov, I. L.: Topology of the high latitude magnetosphere during large magnetic storms and the main mechanisms of relativistic electron acceleration, Adv. Space Res., 43(4), 628633, 2009b.

Delcourt, D. C. and Sauvaud, J.-A.: Populating of the cusp and boundary layers by energetic (hundreds of $\mathrm{keV}$ ) equatorial particles, J. Geophys. Res., 104(A10), 22635-22648, 1999.

DeMichelis, P., Daglis, I. A., and Consolini, G.: Average terrestrial ring current derived from AMPTE/CCE-CHEM measurements, J. Geophys. Res., 102(A7), 14103-14111, 1997.

DeMichelis, P., Daglis, I. A., and Consolini, G.: An average image of proton plasma pressure and of current systems in the equatorial plane derived from AMPTE/CCE-CHEM measurements, J. Geophys. Res., 104(A12), 28615-28624, 1999.

Dubyagin, S. V., Sergeev, V. A., Carlson, C. W., Marple, S. R., Pulkkinen, T. I., and Yahnin, A. G.: Evidence of nearEarth breakup location, Geophys. Res. Lett., 30(6), 1282, doi:10.1029/2002GL016569, 2003.

Frank, L. A. and Sigwarth, J. B.: Findings concerning the position of substorm expansion onsets with auroral images from the Polar spacecraft, J. Geophys. Res., 105(A6), 12747-12761, 2000.

Hori, T., Ohtani, S., Lui, A. T. Y., McEntire, R. W., Maezawa, K., Sato, Y., and Mukai, T. A.: Substorm associated drift echo of energetic protons observed by Geotail: Radial density gradient structure, Geophys. Res. Lett., 30(6), 1330, doi:10.1029/20002GL016137, 2003.

Lui, A. T. Y.: Potential plasma instabilities for substorm expansion onsets, Space Sci. Rev., 113(1), 127-206, 2004.

Lui, A. T. Y. and Hamilton, D. C.: Radial profile of quite time magnetospheric parameters, J. Geophys. Res., 97(A12), 1932519332, 1992.

Lui, A. T. Y., Angelopoulos, V., LeContel, O., Frey, H., Donovan, E., Sibeck, D. J., Liu, W., Auster, H . U., Larson, D., Li, X., Nosé, M., and Fillingim, N. O.: Determination of the substorm initiation region from a major conjunction interval of THEMIS satellites, J. Geophys. Res., 113, A00C04, doi:10.1029/2008JA013424, 2008.

Lyons, L. R., Voronkov, I. O., Donovan, E. F., and Zesta, E.: Relation of substorm breakup arc to other growth-phase auroral arcs, J. Geophys. Res., 107(A11), 1390, doi:10.1029/2002JA009317, 2002.

Millino, A., Orsini, S., and Daglis, I. A.: Empirical model of proton flux in the equatorial inner magnetosphere: Development, J. Geophys. Res., 101(A11), 25713-25729, 2001.
Newell, P. T. and Meng, C.-I.: Mapping the dayside ionosphere to the magnetosphere according to particle precipitation characteristics, Geophys. Res. Lett, 19(6), 609-612, 1992.

Newell, P. T., Sotirelis, T., Ruohoniemi, J. M., Carbary, J. F., Liou, K., Skura, J. P., Meng, C.-I., Deehr, C., Wilkinson, D., and Rich, F. J.: OVATION: Oval variation, assessment, tracking, intensity, and online nowcasting, Ann. Geophys., 20, 1039-1047, 2002, http://www.ann-geophys.net/20/1039/2002/.

Öztürk, M. K. and Wolf, R. A.: Bifurcation of drift shells near the dayside magnetopause, J. Geophys. Res., 112, A07207, doi:10.1029/2006JA012102, 2007.

Shabansky, V. P. and Antonova, A. E.: Topology of particle drift shells in the Earth's magnetosphere, Geomagnetism and Aeronomy, 8, 993-997, 1968 (in Russian).

Shue, J.-H, Chao, J. K., Fu, H. C., Russell, C. T., Song, P., Khurana, K. K., and Singer, H. J.: A new functional form to study the solar wind control of the magnetopause size and shape, J. Geophys. Res., 102(A5), 9497-9511, 1997.

Sitnov, M. I., Tsyganenko, N. A., Ukhorskiy, A. Y., and Brandt, P. C.: Dynamical data-based modeling of the stormtime geomagnetic field with enhanced spatial resolution, J. Geophys. Res., 113, A07218, doi:10.1029/2007JA013003, 2008.

Starkov G. V., Rejenov, V. G., Vorobjev, V. G., Feldstein, Ya. I., and Gromova, L. I.: Structure of auroral precipitations in the daytime sector, Geomagnetism and Aeronomia, 42(1), 186-194, 2002 (in Russian).

Stepanova, M. V., Antonova, E. E., Bosqued, J. M., Kovrazhkin, R. A., and Aubel, K. R.: Asymmetry of auroral electron precipitations and its relationship to the substorm expansion phase onset, J. Geophys. Res., 107(A7), 1134, doi:10.1029/2001JA003503, 2002.

Tsyganenko, N. A.: Global quantitative models of the geomagnetic field in the cislunar magnetosphere for different disturbance levels, Planet. Space Sci., 35(11), 1347-1358, 1987.

Tsyganenko, N. A.: A model of the near magnetosphere with a dawn-dusk asymmetry: 1. Mathematical structure. J. Geophys. Res., 107(A8), 1179, doi:10.1029/2001JA000219, 2002a.

Tsyganenko, N. A.: A model of the near magnetosphere with a dawn-dusk asymmetry: 2. Parameterization and fitting to observations, J. Geophys. Res., 107(A8), 1176, doi:10.1029/2001JA000220, 2002b.

Tsyganenko, N. A. and Sitnov, M. I.: Modeling the dynamics of the inner magnetosphere during strong geomagnetic storms, J. Geophys. Res., 110, A03208, doi:10.1029/2004JA010798, 2005.

Tsyganenko, N. A. and Sitnov, M. I.: Magnetospheric configurations from a high-resolution data-based magnetic field model, J. Geophys. Res., 112, A06225, doi:10.1029/2007JA012260, 2007.

Vorobjev, V. G., Yagodkina, O. I., Starkov, G. V., and Feldstein, Ya. I.: Features of the planetary distribution of auroral precipitation characteristics during substorms, Geomagnetism and Aeronomy, 47(2), 193-204, 2007.

Zou, S., Lyons, L. R., Wang, C.-P., Boudouridis, A., Ruohoniemi, J. M., Anderson, P. C., Dyson, P. L., and Devlin, J. C.: On the coupling between the Harang reversal evolution and substorm dynamics: A synthesis of SuperDARN, DMSP, and IMAGE observations, J. Geophys. Res., 114, A01205, doi:10.1029/2008JA013449, 2009. 